\title{
Comparaison des activités phosphatases acides d'hétérocaryons et d'homocaryons de Suillus granulatus
}

\author{
M. Ducamp ${ }^{\dagger}$ et J.M. Olivier ${ }^{2}$ \\ 1 IRCC-CIRAD, BP 5035, 34032 Montpellier Cedex; \\ 2 INRA, Station de recherches sur les champignons, BP 131, 33140 Pont-de-la-Maye, France
}

(reçu le 10-5-1988, accepté le 20-12-1988)

\begin{abstract}
Résumé - Les activités phosphatases acides du champignon ectomycorhizien Suillus (= Boletus) granulatus sont étudiées sur deux types de matériel : les hétérocaryons (issus de bouturages de carpophores) et les homocaryons (issus de germinations de basidiospores isolées). Leurs activités phosphatases ( $p$-nitrophénylphosphatases, phytases et tripolyphosphatases) sont mesurées à $30,40,50$ et 70 jours, après culture en milieux liquides. On distingue les activités excrétées dans le milieu, les activités accessibles (mesurées sur un thalle intact) et les activités totales (mesurées sur un homogénat de mycélium).

La carence en phosphore entraîne un accroissement des activités phosphatases acides des homocaryons et des hétérocaryons. La culture sur de l'orthophosphate $10 \mu \mathrm{M}$ diminue ces activités de près de $90 \%$. Partant du matériel isolé d'un même carpophore, on constate que les phosphatases acides de l'hétérocaryon sont en général plus actives que celles des homocaryons dérivés. Une bonne corrélation entre les activités phosphatases vis-à-vis de différents substrats a été mise en évidence. Les souches peuvent être classées non seulement selon leurs activités vis-à-vis de ces substrats, mais également d'après leur réponse à un accroissement de la quantité de phosphore disponible dans le milieu. Ces résultats permettent de discuter les perspectives de sélection de souches de $S$. granulatus en vue de la production de carpophores.
\end{abstract}

symbiose mycorhizienne - ectomycorhizes - phosphatases acides - génétique - incompatibilité - Suillus

Summary - Comparison of acid phosphatases in heterocaryotic and homocaryotic strains of Suillus granulatus. Cultivations of heterocaryotic (isolated from fruit-bodies) and homocaryotic mycelia (from monosporic germination) of Suillus granulatus have been carried out in a liquid medium enriched with increasing concentrations of phosphorus. Acid phosphatases have been estimated after 30 to 70 days of growth. Excreted, accessible and total activities have been studied for p-nitropheny/phosphatases, phytases, and tripolyphosphatases enzymes.

A phosphorus deficiency increases the acid-phosphatases activities of homocaryons and heterocaryons. Almost

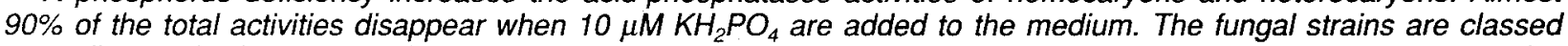
according to the intensity of the observed decrease. Great differrences are also observed between the two strains used to inoculate the trees and the differences are also noted within the homocaryotic or heterocaryotic descendants of these two strains. Heterocaryons are generally more efficient than homocaryons isolated from the same fruit-body. The principles of a strain selection based on phosphatases activities are discussed, the applications for fruit-bodies improvment being chiefly considered.

mycorrhizal symbiosis - ectomycorrhizae - acid phosphatases - genetics - incompatibility - Suillus

\section{Introduction}

La présence d'un symbiote mycorhizien au niveau des racines des arbres s'accompagne souvent d'un effet bénéfique sur le développement de ceux-ci. Une des causes en est l'amélioration de la nutrition minérale de la plante et en particulier celle de l'assimilation du phosphore (Mousain et al., 1978; Mousain et Salsac, 1982; Mousain et Salsac, 1984).

L'accroissement du volume de sol exploré par le système racinaire grâce aux hyphes du symbiote est une des hypothèses avancées pour 
expliquer cette interaction bénéfique. De plus, l'exploitation par le champignon de microsites du sol est possible grâce au faible calibre des hyphes (Callot et al., 1985).

L'accroissement des potentialités enzymatiques de l'association est aussi considéré comme une hypothèse valable. Les phosphatases fongiques interviendraient pour mettre à la disposition de l'hôte du phosphate inorganique à partir des formes insolubles de phosphate (Woolhouse, 1969). Theodorou (1968 et 1971) a démontré que des champignons mycorhiziens (Rhizopogon luteolus (Fr.), Suillus luteus (Fr.) Gray, S. granulatus (Fr. ex L.) O. Kuntze, Cenococcum graniforme (Fr.) sont capables d'utiliser des phytates de calcium et de sodium en libérant une "phytase» dans le milieu de culture. Calleja et d'Auzac (1982) ont comparé in vitro les aptitudes de différentes espèces à assurer la régénération du pool de phosphate soluble du sol à partir de formes organiques.

Chez Suillus (ou Boletus) granulatus, la carence en phosphore du milieu induit une forte augmentation des activités phosphatases acides (Calleja et al., 1980). Parallèlement, Mousain et Salsac (1982), Mousain et al. (1988), ont étudié in vitro les activités phosphatasiques de cette espèce. Cependant, ces travaux ne concernent que des thalles hétérocaryotiques (fructifères) de $S$. granulatus obtenus par bouturage de carpophores.

Dans le cadre d'une étude génétique de l'association S. granulatus - pin maritime, il nous a paru intéressant de comparer les activités phosphatases acides de thalles homocaryotiques et hétérocaryotiques en vue d'une amélioration raisonnée des caractéristiques physiologiques des souches. Un précédent travail avait déterminé les éléments caryologiques et cytologiques nécessaires, précisant en particulier le caractère hétérothallique de l'espèce et le statut nucléaire original des différents types d'hyphes (Ducamp, 1987; Ducamp et al., 1986). Cette recherche s'inscrit dans un programme visant à améliorer le fonctionnement de la symbiose mycorhizienne mais aussi à accroître les perspectives de production de champignons comestibles via la mycorhization contrôlée. Sur ce plan, $S$. granulatus est un bon modèle, puisque la maîtrise de son cycle est acquise "au champ" (Poitou et al., 1983, 1987).

Les résultats rapportés dans cet article concernent plus précisément les activités phosphatases acides (accessibles, excrétées et totales) mises en évidence in vitro, à partir de trois substrats phosphorés chez différents isolats homocaryotiques ou hétérocaryotiques.

\section{Matériel et Méthodes}

\section{Matériel fongique : origine et nomenclature}

Dix-sept thalles sont utilisés au total au cours de ce travail, soit 10 hétérocaryons obtenus par bouturage d'un fragment de carpophore (B) et 7 homocaryons, chacun issu d'une basidiospore unique ( $M$ pour monospore) :

- 2 hétérocaryons, $B 1$ et $B 2$, sont les «souches mères» utilisées à l'origine pour inoculer en conditions contrôlées les pins de la parcelle où sont récoltés tous les carpophores utilisés pour ce travail (Poitou et al., 1983);

- 5 hétérocaryons $\mathrm{B} 1.1, \mathrm{~B} 1.2, \mathrm{~B} 1.3, \mathrm{~B} 1.4, \mathrm{~B} 1.5$ sont issus de bouturages de carpophores récoltés sous des arbres initialement inoculés par B1;

- 3 groupes hétérocaryon-homocaryons dérivés sont constitués par bouturage d'un fragment d'un carpophore (hétérocaryon) et de 2 souches monospores issues du même carpophore (homocaryons), d'où les triplets suivants : $-\mathrm{B} 1.6 \rightarrow \mathrm{M} 1.6 \mathrm{a}$ et $\mathrm{M} 1.6 \mathrm{~b}$ (sous un arbre inoculé à l'origine par $B 1$ ) , $-B 1.7 \rightarrow M 1.7 a$ et $M 1.7 b$ (sous un arbre inoculé à l'origine par $\mathrm{B} 1$ ),$-\mathrm{B} 2.1 \rightarrow \mathrm{M} 2.1 \mathrm{a}$ et M2.1b (sous un arbre inoculé à l'origine par B2);

-1 homocaryon isolé d'un champignon formé sous un arbre inoculé initialement par B1 est inclus également dans cette étude (M1.y).

\section{Cultures mycéliennes}

Les mycéliums sont entretenus sur un milieu de base, gélosé à $1 \%$, contenant $16 \mu \mathrm{M}$ de phosphore minéral (dont $5 \mu \mathrm{M}$ de phosphore apporté par la gélose). Les cultures mères ainsi préparées servent à ensemencer des milieux liquides auxquels $\mathrm{KH}_{2} \mathrm{PO}_{4}$ est ajouté pour obtenir des concentrations de $0,1-10-25$ et $100 \mu \mathrm{M}$ de phosphore inorganique. La quantité de phosphore apportée par les composants du milieu est inférieure à $0,1 \mu \mathrm{M}$; elle peut être considérée comme négligeable bien que le milieu $P=0$ ne puisse être de ce fait considéré comme totalement carencé en phosphore. La composition de base des milieux nutritifs est la suivante : $\mathrm{NaCl} \mathrm{0,2} \mathrm{mM;} \mathrm{KNO}_{3} 2,0 \mathrm{mM} ; \mathrm{NH}_{4} \mathrm{Cl} 1,0 \mathrm{mM}$; $\mathrm{Ca}$ $\mathrm{Cl}_{2}$ 0,5 mM; $\mathrm{MgSO}_{4}, 1,0 \mathrm{mM}$; glucose $55 \mathrm{mM}$; citrate ferrique à $1 \% \quad 0,5 \mathrm{ml}^{-1}$; solution de vitamines 0,1 mil.t-1. Le mélange de vitamines comprend par litre, thiamine $0,5 \mathrm{mg}$; riboflavine $0,5 \mathrm{mg}$; nicotinamide $0,5 \mathrm{mg}$; chlorhydrate de pyridoxine $0,5 \mathrm{mg}$; D-pantothénol $0,5 \mathrm{mg}$. Le $\mathrm{pH}$ du milieu est ajusté à 5,6 avec $\mathrm{NaOH} 0,1 \mathrm{M}$. La culture a lieu à $25^{\circ} \mathrm{C}$, à l'obscurité, sans agitation.

Pour chaque condition expérimentale, 5 flacons contenant $40 \mathrm{ml}$ du milieu choisi sont ensemencés avec un implant mycélien de $8 \mathrm{~mm}$ de diamètre apportant moins de $0,1 \mu \mathrm{M}$ de phosphore. Chaque expérience est répétée 3 à 5 fois; les données analysées portent donc pour chaque traitement au minimum sur 15 mesures élémentaires.

\section{Détermination des activités phosphatasiques}

Les activités suivantes sont déterminées après des temps de culture de $30,40,50$ et 70 jours :

- phosphatases excrétées dans le milieu de culture pendant la croissance mycélienne;

- phosphatases dites "accessibles» (au sens de Calleja et al., 1980), provenant d'un thalle lavé avec une 
solution de glucose (55 mM) puis incubé avec le substrat des enzymes;

- phosphatases dites «totales», libérées par broyage puis homogénéisation du thalle entier et lavées.

Les activités phosphatases sont mesurées en utilisant 3 substrats; 2 composés organiques, le p-nitrophényl phosphate de sodium (PNPP), référence classique du dosage des activités phosphatases, et l'hexophosphate d'inositol ou phytate, généralement bien représenté dans les sols (Cosgrove, 1980a, 1980b), un polyphosphate inorganique, le tripolyphosphate de sodium (TPP) accumulé dans les mycéliums (Martin et al., 1985). Les PNPP-ases sont dosées selon la méthode décrite par Calleja et al. (1980), les TPP-ases et phytases selon Bousquet et al. (1986). Les activités phosphatases sont exprimées en nanomoles du substrat hydrolysé par minute et par gramme de mycélium frais (UG), les teneurs en matières sèches étant relativement constantes $(91 \pm 3 \%)$.

Afin de comparer différents isolats fongiques par leur réaction à une élévation de la teneur en phosphore du milieu, nous définissons 2 indices $i_{1}$ et $i_{100}$ exprimés en \%:

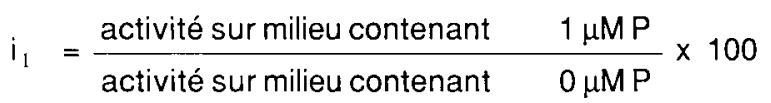

$i_{100}=\frac{\text { activité sur milieu contenant }}{100 \mu \mathrm{MP}}=100$

Ces indices caractérisent donc la diminution d'une activité phosphatasique lorsque la teneur en phosphore du milieu est accrue. Les tests de Student et Newman-Keuls sont appliqués selon Snedecor et Cochran (1984).

\section{Résultats}

Evolution des activités PNPP-ases en fonction du temps de culture

Cultivés sur le milieu contenant $1 \mu \mathrm{M}$ de phosphore inorganique (P1), les homocaryons M1.y et $\mathrm{M} 1.6 \mathrm{~b}$ et les hétérocaryons $\mathrm{B} 1$ et $\mathrm{B} 1.6$ ont des activités excrétées qui augmentent jusqu'au 50 jour de culture (Fig. 1). L'activité de l'hétérocaryon B1.6 est supérieure à celle de l'homocaryon dérivé $\mathrm{M} 1.6 \mathrm{~b}$. Les différences entre souches ne sont cependant plus significatives au seuil de $5 \%$ au-delà du $50^{\circ}$ jour. Il en est de même pour les PNPP-ases accessibles (Fig. 2), dont les fluctuations d'activité au cours du temps sont retrouvées régulièrement. Une durée de culture de 40 jours est discriminante et sera retenue pour les comparaisons de souches.

\section{Influence des teneurs en phosphore des milieux sur les activités PNPP-ases}

Dans une première série d'essais, l'intensité des activités PNPP-ases diminue avec des concentrations croissantes en phosphore dans le milieu;

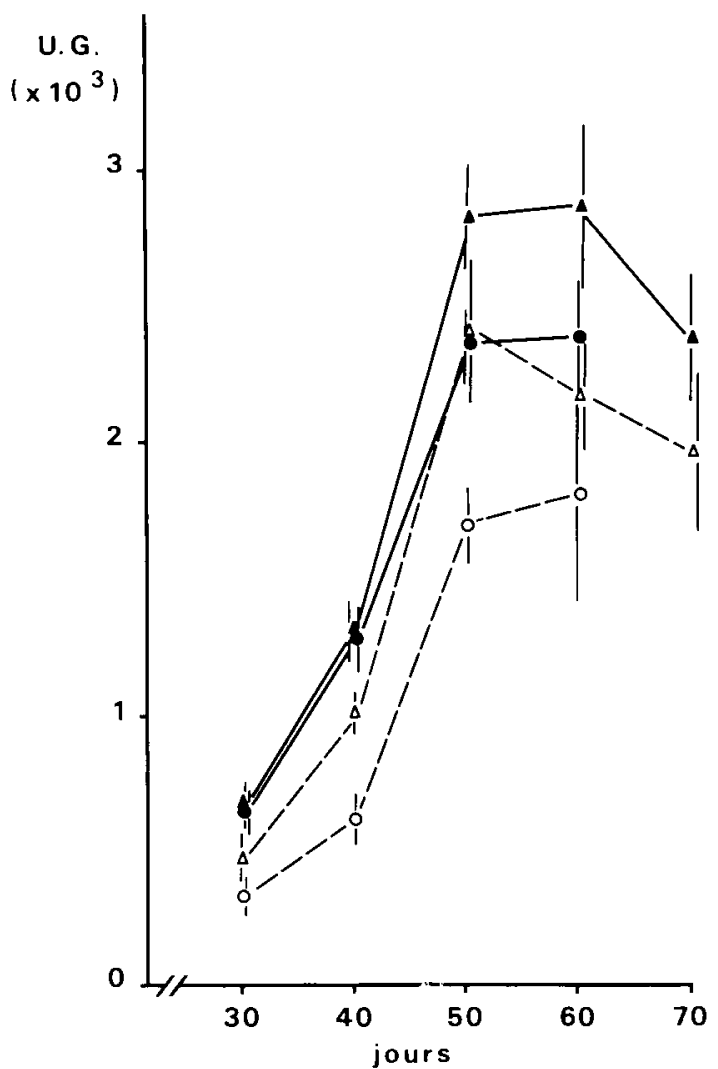

Fig. 1. Evolution des activités PNPP-ases excrétées de 2 homocaryons et 2 hétérocaryons en fonction du temps de culture sur milieu contenant $1 \mu \mathrm{M}$ de phosphore. (UG = nmoles de P.N.P.P. hydrolysées $\times \mathrm{mn}^{-1} \times \mathrm{g}^{-1}$ ). $\longrightarrow \mathrm{B} 1$, $\curvearrowright \mathrm{M} 1 . \mathrm{y}, \Delta \longrightarrow \Delta \mathrm{B} 1.6, \Delta-\Delta \mathrm{M} 1.6 \mathrm{~b}$.

U.G.

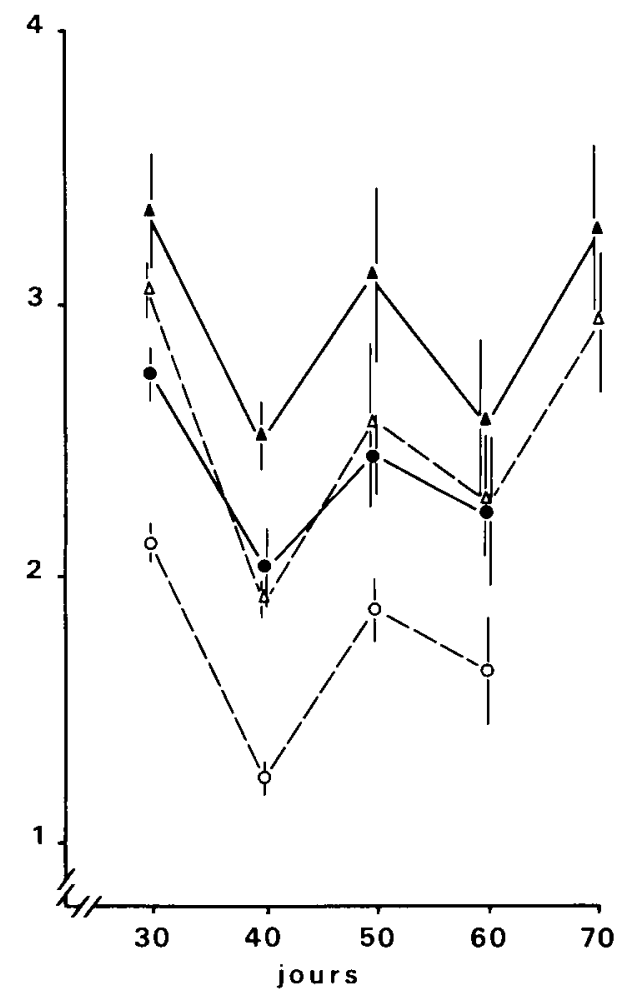

Fig. 2. Evolution des activités PNPP-ases accessibles de 2 homocaryons et 2 hétérocaryons en fonction du temps de culture sur milieu contenant $1 \mu \mathrm{M}$ de phosphore. (UG : voir légende Fig. 1). $\longrightarrow \mathrm{B} 1, \mathrm{O}-\mathrm{OM} 1 . \mathrm{y}, \mathbf{\Delta} \longrightarrow \mathrm{B} 1.6$, $\Delta--\Delta \mathrm{M} 1.6 \mathrm{~b}$. 
pour une concentration de $10 \mu \mathrm{M}$, la réduction est d'environ $90 \%$ (Figs. 3 et 4). L'effet du phosphore est du même ordre pour l'hétérocaryon et l'homocaryon, bien que les valeurs de leurs activités respectives soient significativement différentes pour les concentrations de 0 et $1 \mu \mathrm{M}$ de phosphore.

Le même type de dosage a été repris avec douze isolats, après culture sur des milieux contenant 0,1 et $100 \mu \mathrm{M}$ de phosphore. Les précédentes observations sur l'effet inhibiteur des doses croissantes de phosphore sont confirmées (Tabl. I).

Les indices $i_{1}$ et $i_{100}$ précédemment définis facilitent la comparaison entre souches, du moins en ce qui concerne $i_{1}$ et les PNPP-ases excrétées (Tabl. II). L'indice $i_{100}$ traduit l'homogénéité

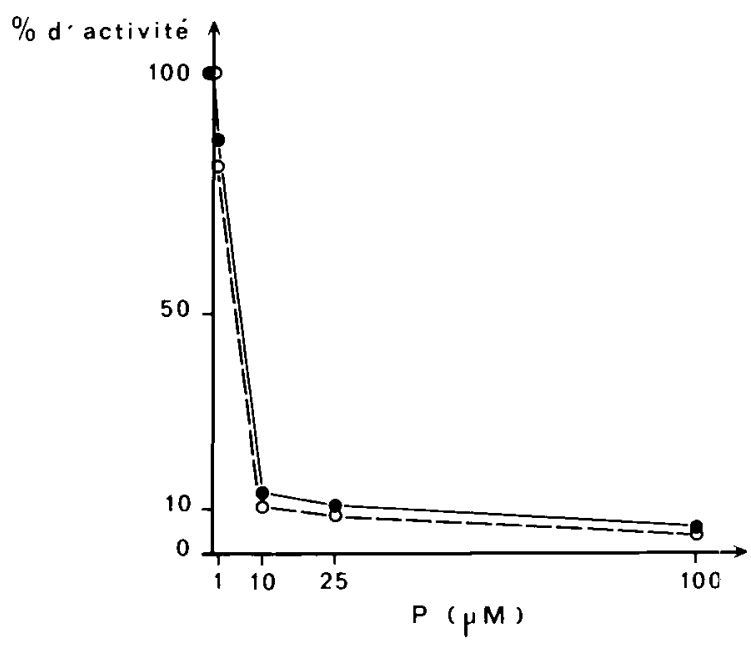

Fig. 3. Evolution des activités PNPP-ases excrétées d'un homocaryon et d'un hétérocaryon en fonction de la concentration en phosphore du milieu. $\longrightarrow \mathrm{B} 1, \mathrm{O} \longrightarrow \mathrm{M} 1 . \mathrm{y}^{\prime}$.

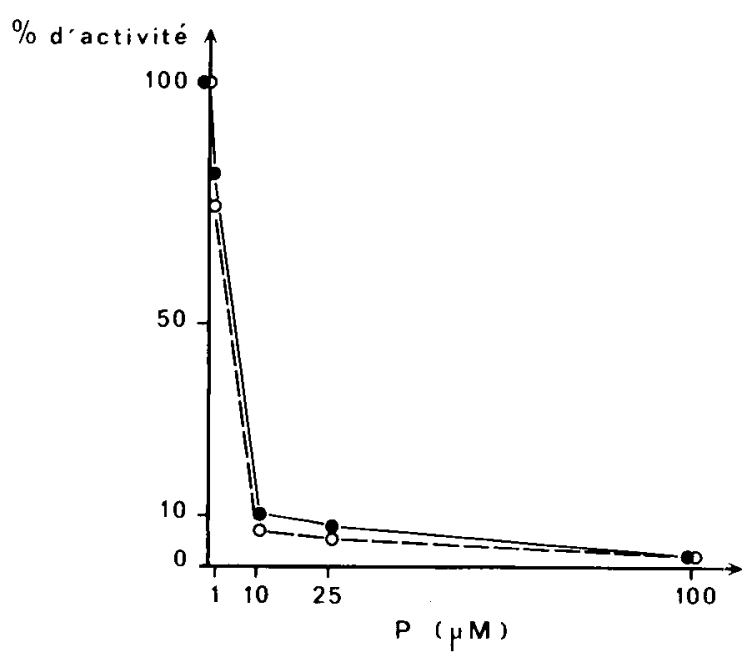

Fig. 4. Evolution des activités PNPP-ases accessibles c'un homocaryon et d'un hétérocaryon en fonction de la concentration en phosphore du milieu. $\longrightarrow \mathrm{B} 1, \mathrm{O} \longrightarrow \mathrm{M} 1 . \mathrm{y}$. des réponses des différents isolats à une forte augmentation des concentrations de phosphore disponible. Avec $i_{1}$, la réduction constatée au niveau des PNPP-ases accessibles semble assez homogène, quelle que soit la souche. Les variations d'activités PNPP ases excrétées sont significativement différentes selon les souches et permettent de distinguer 3 groupes d'isolats :

- faible inhibition par $1 \mu \mathrm{M} P(<20 \%)$ pour la souche d'origine B1, le groupe constitué par l'hétérocaryon B1.6 et les homocaryons dérivés M1.6a et M1.6b;

- inhibition moyenne par $1 \mu \mathrm{M} \mathrm{P}(20$ à $35 \%)$ pour l'hétérocaryon $\mathrm{B} 1.7$ et les homocaryons dérivés M1.7a et M1.7b, ainsi que l'homocaryon M1.y (dérivé de $\mathrm{B} 1$ après trois cycles de fructification);

- inhibition forte par $1 \mu \mathrm{M} \mathrm{P} \mathrm{(>35 \% )} \mathrm{pour} \mathrm{l'autre}$ souche d'origine, B2, l'hétérocaryon B2.1 (descendant de B2) et les homocaryons dérivés M2.1a et M2.1b.

Les activités des souches d'origine B2 sont les plus fortement réduites par le phosphore; elles sont aussi inférieures aux activités des souches d'origine B1. Parmi celles-ci, aucune corrélation entre l'indice d'inhibition par le phosphore $\left(i_{1}\right)$ et l'activité PNPP-ase excrétée (exprimée en UG) n'a été mise en évidence. La souche M1.6a est peu inhibée $\left(i_{1}=9,1 \%\right)$ mais elle présente des activités PNPP-ases élevées, 813 UG sur milieu $P=1 \mathrm{M}$ et $884 \mathrm{UG}$ sur $\mathrm{P}=0$ ). A l'opposé, la souche M1.7a est plus fortement inhibée $\left(i_{1}=32,5 \%\right)$ mais son activité est faible (795 UG) sur milieu $P=1 \mathrm{M}$ et plus forte sur milieu $P=0$ (1 $325 \cup G$ ).

Une relation entre indices $i_{1}$ (ou $i_{100}$ ) et croissance des thalles a été recherchée; les mesures de la production mycélienne au $40^{\circ}$ jour de culture en milieux liquides permettent de calculer les rapports entre biomasses produites en présence de différentes concentrations de phosphore (pour l'ensemble des souches).

$$
\begin{aligned}
& -\mathrm{C}_{1}=\frac{\text { biomasse en présence de }}{\text { bímasse en présence de }} \frac{1 \mu \mathrm{MP}}{0 \mu \mathrm{MP}}=2,25 \pm 0,25
\end{aligned}
$$

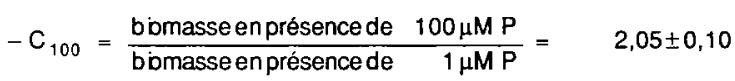

On remarque la faible variabilité au sein des données (calculs sur 25 mesures/souche); on peut conclure à une réponse identique de tous les isolats à une augmentation de la teneur en phosphore, en ce qui concerne l'accroissement de biomasse.

Aucune corrélation entre les indices $i_{1}$ (ou $i_{100}$ ) et $C_{1}$ (ou $C_{100}$ ) ne peut être définie; par exemple, les souches pour lesquelles l'activité est peu inhibée par $\mathrm{P}\left(\mathrm{i}_{1}<20 \%\right)$ ont des indices de croissance 
Tableau I. Comparaison des activités PNPP-ases sur 3 milieux contenant 0,1 ou $100 \mu \mathrm{M}$ de phosphore (résultats exprimés en $U G=n$ mol de substrat hydrolysé. $\min ^{-1} \cdot g^{-1} \mathrm{mf}$ ).

PNPP-ases excrétées

$P=0 \quad P 1 \quad P 100$

$1414 \pm 130$

$834 \pm 54$

$1446 \pm 90$

$884 \pm 91$

M1.6a

M1.6b

B1.7

M1.7a

M1.7b

B2

B2.1

M2.1a

M2.1b
$1178 \pm 65$

$1938 \pm 100$

$1325 \pm 69$

$1396 \pm 71$

$1278 \pm 61$

$1221 \pm 49$

$922 \pm 53$

$1182 \pm 64$

$\begin{array}{rr}1292 \pm 122 & 25 \pm 4 \\ 610 \pm 52 & 18 \pm 6 \\ 1290 \pm 108 & 26 \pm 5 \\ 813 \pm 64 & 12 \pm 4 \\ 1003 \pm 58 & 14 \pm 5 \\ 1426 \pm 115 & 25 \pm 3 \\ 795 \pm 73 & 11 \pm 3 \\ 1015 \pm 62 & 12 \pm 3 \\ 728 \pm 64 & 10 \pm 3 \\ 738 \pm 44 & 9 \pm 3 \\ 615 \pm 51 & 10 \pm 2 \\ 705 \pm 40 & 10 \pm 3\end{array}$

$1292 \pm 122$

$18 \pm 6$

$2361 \pm 134$

$1603 \pm 75$

$2722 \pm 129$

$2215 \pm 75$

$2328 \pm 97$

$1675 \pm 99$

$2089 \pm 80$

$2246 \pm 75$

$1743 \pm 91$

$1948 \pm 103$

$1497 \pm 73$

$1538 \pm 79$
$2023 \pm 153$

$1250 \pm 67$

$2506 \pm 141$

$1841 \pm 72$

$1905 \pm 64$

$2458 \pm 106$

$1824 \pm 81$

$1921 \pm 48$

$1464 \pm 78$

$1717 \pm 75$

$1322 \pm 64$

$1375 \pm 84$

P100

$116 \pm 15$

$73 \pm 12$

$117 \pm 21$

$92 \pm 13$

$95 \pm 14$

$129 \pm 17$

$96 \pm 14$

$102 \pm 18$

$83 \pm 12$

$101 \pm 13$

$84 \pm 19$

$92 \pm 12$

Tableau II. Mesure des indices $i_{1}$ et $i_{100}$ caractérisant l'inhibition des activités PNPP-ases excrétées et accessibles par des concentrations croissantes en phosphore dans le milieu. (Seuil de signification selon le test de Newman-Keuls, $P=0,05$.)

PNPP-ases excrétées

$i_{1}$
PNPP-ases accessibles

$i_{1} \quad i_{100}$

\begin{tabular}{lrlllrlrl} 
B1 & 18,7 & ab & 98,1 & a & 14,4 & ab & 94,3 & a \\
M1.y & 26,9 & abc & 97,1 & a & 22,0 & b & 94,2 & a \\
B1.6 & 11,8 & a & 98,0 & a & 8,0 & a & 95,3 & a \\
M1.6a & 9,1 & a & 98,6 & a & 16,9 & b & 95,0 & a \\
M1.6b & 14,9 & a & 98,6 & a & 18,2 & b & 95,0 & a \\
B1.7 & 26,5 & ab & 98,3 & a & 8,2 & a & 94,8 & a \\
M1.7a & 32,5 & b & 98,6 & a & 12,7 & ab & 94,8 & a \\
M1.7b & 27,3 & ab & 98,8 & a & 14,5 & ab & 94,7 & a \\
B2 & 43,0 & c & 98,6 & a & 16,0 & ab & 94,3 & a \\
B2.1 & 39,6 & c & 98,8 & a & 11,9 & ab & 94,2 & a \\
M2.1a & 35,3 & bc & 98,4 & a & 11,7 & ab & 93,7 & a \\
M2.1b & 40,4 & c & 98,6 & a & 10,7 & ab & 94,0 & a \\
\hline
\end{tabular}

$\mathrm{C}_{1}$ variant de 1,75 à 2,56. A l'opposé, les souches dont l'activité enzymatique est fortement inhibée $\left(i_{1}>35 \%\right)$ ont des indices $C_{1}$ variant de 2 à 2,57 . On peut donc conclure que l'effet inhibiteur du phosphore exercé sur les activités phosphatases est spécifique et non lié à une perturbation générale du développement du mycélium qui est au contraire stimulé par un apport croissant de phosphore.

Les résultats rapportés dans les Tableaux I et II mettent en évidence une différence nette de comportement entre les deux souches hétérocaryotiques $\mathrm{B} 1$ et $\mathrm{B} 2$ utilisées pour les mycorhizations d'origine. Cette différence persiste après plusieurs cycles de fructification et peut être retrouvée dans les descendants qu'ils soient homo ou hétérocaryotiques. On note aussi que les activités PNPP-ases excrétées ou accessibles des homocaryons sont significativement inférieures $(P=0,05)$ à celles des hétérocaryons, sauf en ce qui concerne B2.1 et ses dérivés M2.1a et M2.1b, dont il faut souligner les faibles niveaux d'activités. A travers les différents résultats, la concentration de $1 \mu \mathrm{M}$ de phosphore parait discriminante (au contraire de 10 ou 100 $\mu \mathrm{M})$; elle n'entraîne aucun problème d'interprétation lié à du phosphore "parasite", comme ce serait le cas avec le $0 \mu \mathrm{M}$ théorique. Elle est donc retenue pour la suite des essais. 
Comparaison des souches en fonction des différentes activités phosphatases

Les 12 isolats sont comparés pour leurs activités PNPP-ases, TPP-ases et phytases après 40 jours de culture sur le milieu contenant $1 \mu \mathrm{M}$ de phosphore (Tabl. III). Comme les écarts entre les souches d'origine $\mathrm{B} 1$ et $\mathrm{B} 2$, les différences entre hétérocaryons et homocaryons sont en majorité significatives. Seules les phytases excrétées (origines B1 et B2) et les TPP ases excrétées (origine $\mathrm{B} 2$ ) font exception à cette règle.

Les faibles activités du matériel fongique dérivé de B2 sont confirmées pour les différents types d'activités. Les Figures 5 (a, b, c, d) et 6 (a, $b, c)$ reprennent les données en les décomposant par groupes de souches et ceci par rapport à un indice 100 défini par les activités de l'isolat B1.7 considéré comme le plus performant. Les écarts entre un hétérocaryon et ses homocaryons dérivés sont nets pour les triplets d'origine B1. De la Figure 5d ressortent de grandes différences entre hétérocaryons, ce qui témoigne pour les souches d'origine B1 d'une évolution au cours des cycles successifs de fructification. Les écarts sont particulièrement marqués pour les phytases excrétées pour lesquelles on ne retrouve que deux groupes, l'un avec les activités proches de B1.7 (75 à 105\%), l'autre avec des activités faibles (20 à $30 \%$ de celles de la référence $\mathrm{B} 1.7)$. L'analyse par triplets hétérocaryonhomocaryons pour les différentes activités excrétées permet de situer les écarts (Figs. $5 \mathrm{a}, \mathrm{b}, \mathrm{c}$ ).

Cependant, le classement des souches diffère peu d'une activité à l'autre, d'où l'hypothèse d'une corrélation entre les différentes activités :
- corrélation entre PNPP-ases :

- accessibles vs excrétées, $r=0,92(P=0,01)$,

- accessibles vs totales, $r=0,97(P=0,01)$,

- excrétées vs totales, $\quad \mathrm{r}=0,97(P=0,01)$;

- corrélation entre PNPP-ases et phytases :

- excrétées, $\mathrm{r}=0,75(P=0,01)$,

- totales, $r=0,61(P=0,05)$;

- corrélation entre PNPP-ases et TPP-ases,

- excrétées, $r=0,75(P=0,05)$;

- corrélation entre phytases et TPP-ases,

- excrétées, $\mathrm{r}=0,69(P=0,05)$.

Les corrélations entre les PNPP-ases sont bonnes et permettent de conclure que la détermination des activités excrétées a une bonne probabilité de refléter aussi le classement des souches pour les PNPP-ases totales ou excrétées. Les corrélations avec les autres activités sont moins bonnes et ne peuvent être prises en compte qu'en première approximation. La variabilité plus importante des activités TPP-ases et phytases est un résultat qu'il conviendra d'exploiter avec une gamme plus large de souches.

\section{Discussion}

Dans les conditions des essais, il est confirmé que Suillus granulatus est une espèce à fortes activités phosphatases en milieu acide, comme l'avaient précédemment montré Calleja et al. (1980), Calleja et d'Auzac (1982), Bousquet et al. (1986a, 1986b), Ducamp et al. (1986).

Les corrélations entre différents types d'activités phosphatases acides paraissent claires; ce

Tableau III. Comparaison des différentes activités phosphatases acides des hétérocaryons et homocaryons en fonction de la souche fongique B1 ou B2 utilisée à l'origine pour la mycorhization des arbres. Valeurs exprimées en UG = nmol de substrat hydrolysé $\cdot \mathrm{mn}^{-1} \cdot \mathrm{g}^{-1} \mathrm{mf}(\star, *, \hat{\mathrm{n}}=$ différences non significatives au seuil $P=0,05)$.

\begin{tabular}{|c|c|c|c|c|}
\hline \multirow[t]{2}{*}{ Activités } & \multicolumn{2}{|c|}{$\begin{array}{c}\text { Origine } \\
\qquad 1\end{array}$} & \multicolumn{2}{|c|}{$\begin{array}{c}\text { Origine } \\
\quad B 2\end{array}$} \\
\hline & Hétérocaryons & Homocaryons & Hétérocaryons & Homocaryons \\
\hline \multicolumn{5}{|l|}{ PNPP -ases } \\
\hline - excrétées & $912 \pm 159$ & $641 \pm 123$ & $770 \pm 324$ & $537 \pm 61$ \\
\hline - accessibles & $2163 \pm 141$ & $1621 \pm 157$ & $1476 \pm 112$ & $1290 \pm 46$ \\
\hline - totales & $9502 \pm 541$ & $5768 \pm 803$ & $7665 \pm 613$ & $6087 \pm 682$ \\
\hline \multicolumn{5}{|l|}{ Phytases } \\
\hline - excrétées & $0,692 \pm 0,218$ & $0,544 \pm \pm 0,135$ & $0,260_{*} \pm 0,021$ & $0,190_{*} \pm 0,043$ \\
\hline - totales & $264 \pm 24$ & $206 \pm 29$ & $161 \pm 41$ & $153 \pm 22$ \\
\hline TPP ases & & & & \\
\hline - excrétées & $9,50 \pm 1,82$ & $6,24 \pm 1,91$ & $2,55_{\text {方 }} \pm 0,17$ & $2,41_{\text {凸 }} \pm 0,44$ \\
\hline
\end{tabular}




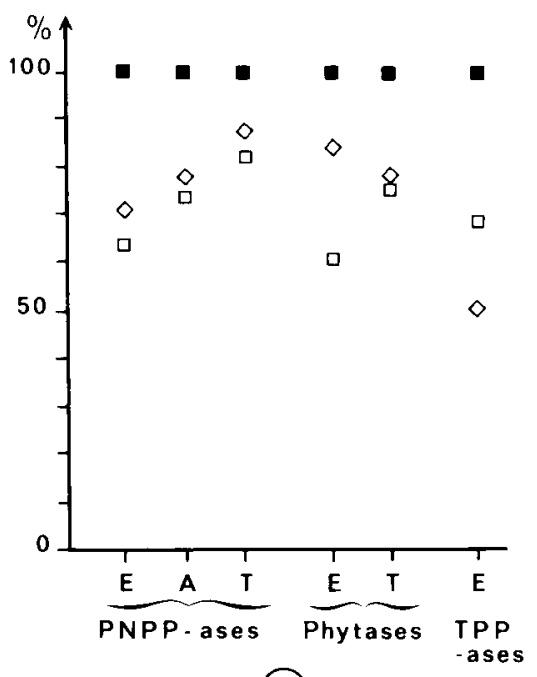

(a)

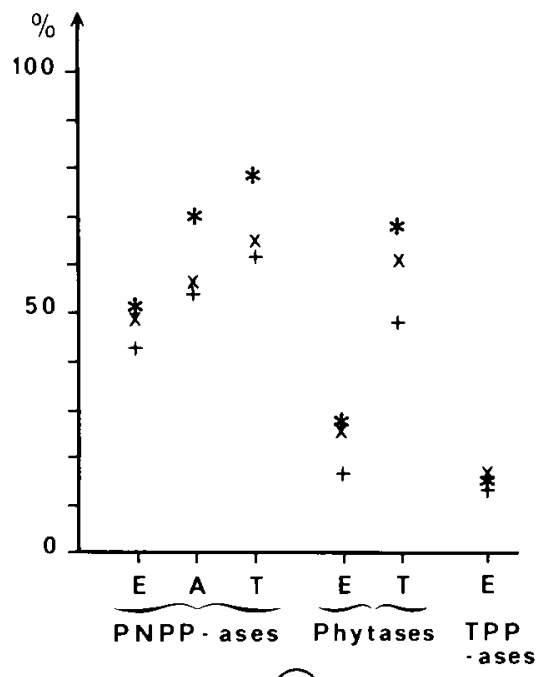

(c)

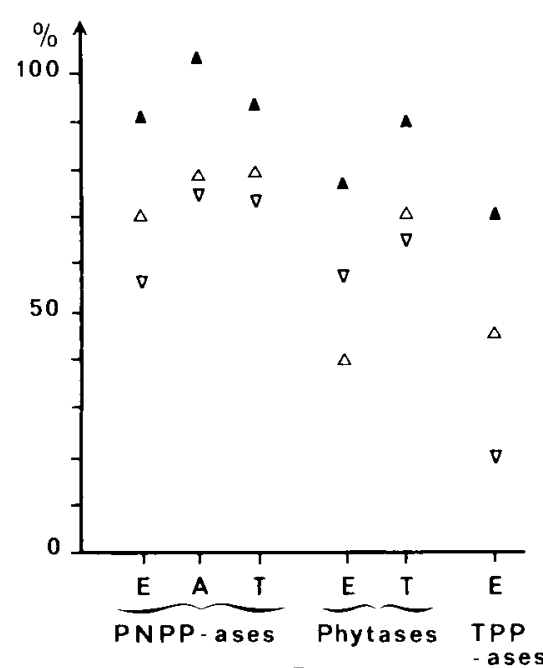

(b)

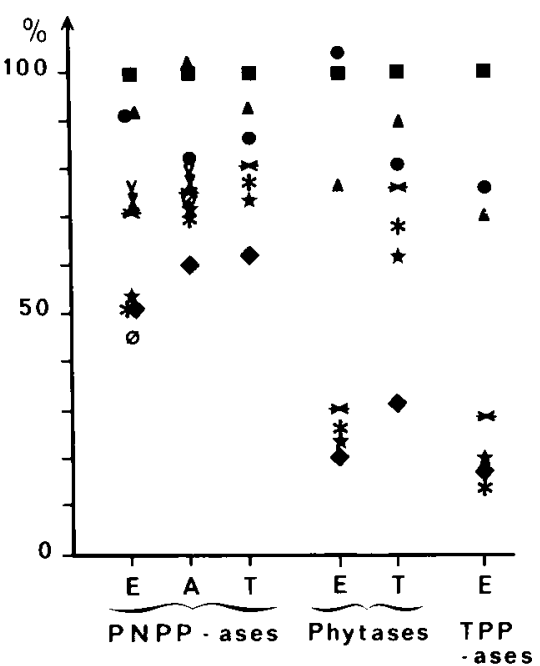

(d)

Fig. 5. Comparaison sur milieux contenant $1 \mu \mathrm{M}$ de phosphore des différentes activités phosphatases acides de 8 hétérocaryons et 6 homocaryons (valeurs exprimées en \% de l'activité de la souche B1.7). E : excrétées, A : accessibles, T : totales.

a.- Triplet B1.7 et homocaryons dérivés M1.7a et M1.7b; b.- Triplet B1.6 et homocaryons dérivés M1.6a et M1.6b; c.- Triplet B2.1 et homocaryons dérivés $\mathrm{M} 2.1 \mathrm{a}$ et $\mathrm{M} 2.1 \mathrm{~b} ; \mathrm{d}$.- Comparaison des hétérocaryons entre eux.

$\bullet \mathrm{B} 1, \diamond \mathrm{B} 2, \star \mathrm{B} 1.1, \varnothing \mathrm{B} 1.2, \mathrm{X} \mathrm{B} 1.3, \mathrm{YB} 1.4, * \mathrm{~B} 1.5, \Delta \mathrm{B} 1.6, \nabla \mathrm{M} 1.6 \mathrm{a}, \Delta \mathrm{M} 1.6 \mathrm{~b}, \square \mathrm{B} 1.7, \square \mathrm{M} 1.7 \mathrm{a}, 0 \mathrm{M} 1.7 \mathrm{~b}, * \mathrm{~B} 2.1,+\mathrm{M} 2.1 \mathrm{a}, \mathrm{X}$ M2.1b.

résultat généralise celui obtenu par Mousain et al. (1988) avec une autre souche de S. granulatus.

Les souches peuvent être comparées d'après leurs activités enzymatiques en situation de carence, par exemple en présence de $1 \mu \mathrm{M}$ de phosphore; on met ainsi en évidence de fortes différences entre thalles issus de boutures (hétérocaryons) et thalles issus de la germination d'une seule spore (homocaryons). L'indice $i_{1}$, défini au cours de ce travail, représente un autre critère de comparaison, qui, malgré le faible nombre des souches étudiées, semble indépendant du précédent; $i$, caractérise l'aptitude d'un thalle à conserver une activité enzymatique lorsque la teneur en phosphore du milieu s'accroît.

Les hétérocaryons ont des activités enzymatiques supérieures à celles des homocaryons dérivés, ce qui rejoint les résultats de Wagner et al. (1988) sur l'activité glutamate déshydrogénase d'Hebeloma cylindrosporum. Avec le même champignon, Gay et Debaud (1987) notent que la production d'acide indol-acétique par certains homocaryons est, contrairement à l'exemple précédent, supérieure à celle du dicaryon d'origine. Les caractères intéressants pour la valorisation pratique des symbioses mycorhiziennes sont 


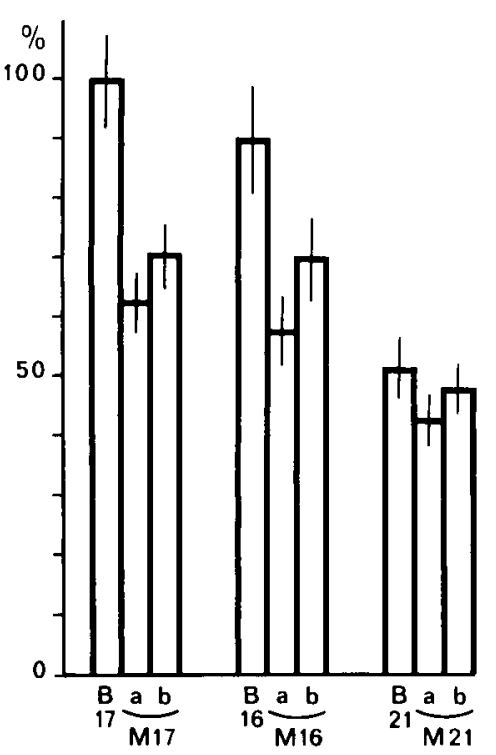

PNPP-ases excrétées

(a)

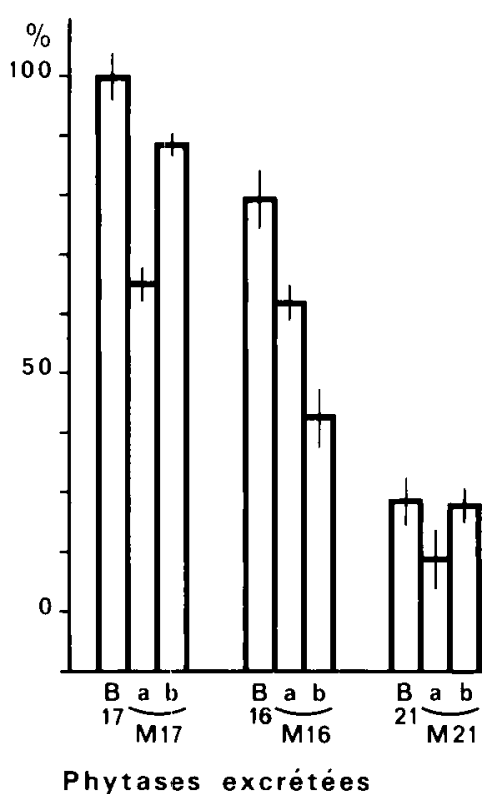

(b)

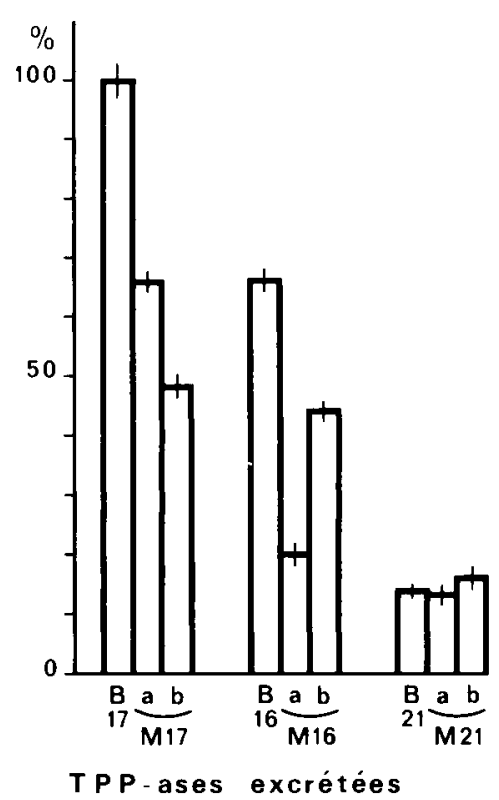

(c)

Fig. 6. Voir légende de la Figure 5, mais avec regroupement par activités excrétées et par triplets hétérocaryon - homocaryons dérivés.

donc sous contrôle génique complexe. Pour chaque cas, il sera nécessaire de mettre en œuvre des méthodes de génétique quantitative (Falconer, 1981), pour lesquelles existent peu d'exemples d'applications à des champignons supérieurs en dehors des modèles classiques, Coprinus ou Schizophyllum. Outre les travaux déjà rapportés pour Hebeloma, on peut citer l'analyse de l'hérédité des caractères de fructification réalisée par Marmeisse (1987) avec Agrocybe aegerita. Au-delà des perspectives d'amélioration des souches utilisées pour la mycorhization, s'ouvrent des possibilités d'étude des populations selon le schéma défini par Guillaumin (1986) pour Armillaria mellea. Avec $S$. granulatus, un tel travail est maintenant possible depuis la mise en évidence du système d'incompatibilité sexuelle chez cette espèce (Ducamp, 1987). La récupération d'hétérocaryons "contrôlés", peut se faire grâce à des techniques accroîssant la fréquence des boucles d'anastomoses, généralement rares in vitro chez S. granulatus (Mamoun et al., non publié).

Dans un tel contexte, la valeur des critères liés aux phosphatases peut être discutée; plusieurs auteurs ont montré l'intérêt pour la plante d'une forte activité phosphatasique au niveau des mycorhizes (Berjaud et d'Auzach, 1986). Dans notre cas, il est difficile d'établir une corrélation entre activité in vitro et comportement "au champ». Poitou et al. (1987), mesurant le rendement en carpophores, constatent que les arbres mycorhizés par B2 sont significativement moins productifs que les arbres mycorhizés par B1. Cependant aucune différence de taille ou de teneurs en phosphore des aiguilles n'a été observée entre plantes mycorhizés par B1 ou B2, malgré la carence et l'appauvrissement du sol en phosphore assimilable $(P=2,47$ meq p. $100 \mathrm{~g}$ m.s. en 1980 et 1,16 meq en 1983, dosage à l'acide fluorhydrique, Soignet, comm. pers.). L'interprétation de ces résultats est difficile, les arbres mycorhizés étant issus de semis et donc génétiquement hétérogènes. Toute corrélation entre les tests in vitro et l'activité "au champ» ne peut donc être recherchée que dans un dispositif plus rigoureux, mais aussi plus laborieux, faisant appel au clonage de l'hôte et du champignon.

En se limitant aux observations déjà réalisées, l'analyse des phosphatases met en évidence de fortes différences entre les hétérocaryons isolés de carpophores récoltés sous des arbres associés initialement à $\mathrm{B} 1$. Une évolution a donc eu lieu au cours des cycles successifs de fructification; elle pose le problème de la pérennité de la souche utilisée à l'origine pour la mycorhization contrôlée. Une souche clonée et "sélectionnée" subira au cours des années une évolution conduisant à une population mycorhizienne, évolution dépendant à la fois des variations de la souche d'origine et d'une possible recombinaison avec des éléments sauvages de la même espèce. A l'opposé de cet aspect négatif, les résultats montrent que la différence entre B1 et B2 s'est globalement maintenue; l'avantage initial de B1 se retrouve après 5 années de fructification. 
En conclusion, la mycorhization contrôlée du pin maritime avec les bolets offre des perspectives intéressantes d'application, mais nécessite une maîtrise du matériel fongique et du végétal associé. La sélection des champignons impose un travail approfondi de génétique quantitative, centré sur l'hérédité de potentialités biologiques majeures, parmi lesquelles les activités enzymatiques. L'introduction récente de l'approche immuno-enzymatique par Bousquet (1987) constitue un apport important pour un criblage plus rapide des aptitudes des souches.

II reste à établir la corrélation entre ces critères de sélection en laboratoire et les objectifs motivant l'amélioration, pour nous une production élevée et régulière de carpophores.

\section{Remerciements}

Les auteurs expriment leur gratitude à D. Mousain et M. Mamoun pour l'aide apportée à la conception et à la réalisation de ce travail soutenu par un AIP INRA $n^{\circ} 4471$. Ils rendent hommage à J. Delmas et N. Poitou qui sont à l'origine de la domestication de Suillus granulatus.

\section{Références}

Berjaud C. \& d'Auzac J. (1986) Isolement et caractérisation des phosphatases d'un champignon ectomycorhizogène typique, Pisolitus tinctorius. Effet de la carence en phosphate. Physiol. Veg. 24, 2, 163-172

Bousquet N. (1987) Etude immuno-enzymatique de phosphatases acides de champignons ectomycorhiziens. Thèse Doct. USTL, Montpellier

Bousquet N., Mousain D. \& Salsac L. (1986a) Influence de l'orthophosphate sur les activités phosphatases acides de Basidiomycètes ectomycorhiziens. Physiol. Vég. 24, 2, 153-162

Bousquet N., Mousain D. \& Salsac L. (1986b) Use Of phytate by ectomycorrhizal fungi. In: Physiological and Genetical Aspects of Mycorrhizae. (GianinazziPearson and Gianinazzi, eds.) INRA, Paris, pp. 363368

Calleja M. \& d'Auzac J. (1982) Carence phosphatée et phosphatases : comparaison de champignons mycorhiziens et saprophytes. In: Les Mycorhizes : Biologie et Utilisation. Colloq. INRA 13, INRA, Paris, pp. 107112

Calleja M., Mousain D., Lecouvreur B. \& d'Auzac J. (1980) Influence de la carence phosphatée sur les activités phosphatases acides de trois champignons mycorhiziens : Hebeloma edurum Metrod., Suillus granulatus (Fr. ex. L.) O. Küntze et Pisolithus tinctorius (Pers.) Coker et Couch. Physiol. Vég. 18, 3, 489504

Callot G., Mousain D. \& Plassard C. (1985) Concentrations de carbonate de calcium sur les parois des hyphes mycéliens. Agronomie 5, 2, 143-150

Cosgrove D.J. (1980a) Inositol phosphates in soils and sediments. In: Inositol Phosphates. Their Chemistry, Biochemistry and Physiology. (D.J. Cosgrove, ed.) Elsevier Sci. Publ. Co., Amsterdam, Oxford, New York, pp. 128-138

Cosgrove D.J. (1980b) Inositol hexakisphosphates. In: Inositol Phosphates. Their Chemistry, Biochemistry and Physiology. (D.J. Cosgrove, ed.) Elsevier, Amsterdam, Oxford, New York, pp. 26-43

Ducamp M. (1987) Contributions à l'étude d'un champignon ectomycorhizien : Suillus granulatus Fr. ex. L. Thèse $3^{\mathrm{e}}$ cycle, Bordeaux II

Ducamp M., Poitou N. \& Olivier J.M. (1986) Comparaison cytologique et biochimique entre cultures monospores et boutures de carpophores chez Suillus granulatus. In: Physiological and Genetical Aspects of Mycorrhizae. (Gianinazzi-Pearson and Gianinazzi, eds.) INRA, Paris, pp. 575-588

Falconer D.S. (1981) Introduction to Quantitative Genetics. 2nd edition, Longman ed, Harlow

Gay G. \& Debaud J.C. (1987) Genetic study on indole3-acetic acid production by ectomycorrhizal Hebeloma species : inter- and intra-specific variability in homo- and dikaryotic mycelia. Appl. Microbiol. Biotechnol. 26, 141-146

Guillaumin J.J. (1986) Contribution à l'étude des Armillaires phytopathogènes, en particulier du groupe Mellea. Thèse d'Etat, Université Lyon

Marmeisse R. (1987) Etude des cycles sexuel et parasexuel du Basidiomycète supérieur Agrocybe aegerita. Thèse Doct. Univ. Claude-Bernard, Lyon

Martin F., Marchal J.P., Timinska A. \& Canet D. (1985) The metabolism and physical state of polyphosphate in ectomycorrhizal fungi. A ${ }^{31} \mathrm{P}$ nuclear magnetic resonance study. New Phytol. 101, 275-290

Mousain D. \& Salsac L. (1982) Nutrition phosphatée et activités phosphatases acides des symbiotes ectomycorhiziens cultivés isolément ou en association. In: Les Mycorhizes : Biologie et Utilisation. Colloq. INRA 13, éd. INRA, Paris, pp. 87-95

Mousain D. \& Salsac L. (1984) Effet du phosphate et du calcium sur la croissance et l'accumulation ionique chez les champignons ectomycorhiziens en culture in vitro. Can. J. Bot. 62, 2600-2609

Mousain D., Poitou N. \& Delmas J. (1978) La symbiose mycorhizienne; résultats obtenus avec l'Hebeloma cylindrosporum et le pisolithus tinctorius; perspectives d'application agronomique. Mushroom Sci. X, I, 949956

Mousain D., Bousquet N. \& Polard C. (1988) Comparaison des activités phosphatases d'Homobasidiomycètes ectomycorhiziens en culture in vitro. J. Eur. Pathol. For. 18, 299-309

Poitou N., Mamoun M., Ducamp M. \& Delmas J. (1983) Premiers résultats de fructification in situ d'un bolet mycorhizien comestible en conditions contrôlées et espérance pour la culture des cèpes nobles. PHM 239, 45-47

Poitou N., Mamoun M., Ducamp M., Guinberteau J. \& Olivier J.M. (1987) Mycorhization contrôlée et culture expérimentale au champ de Boletus (= Suillus) granulatus et Lactarius deliciosus. Mushroom Sci. XII (à paraître)

Snedecor G.W. \& Cochran W.G. (1984) Méthodes statistiques. ACTA, Paris

Theodorou C. (1968) Inositol phosphate in needles of 
Pinus radiata and the phytase activity of mycorhizal fungi. Trans. 9th. Intern. Congres. Soil Sci. 3, 483-490

Theodorou C. ( $\$ 971)$ The phytase activity of the mycorrhizal fungus Rhizopogon luteolus. Soil Biol. Biochem. 3, 89-90

Wagner F., Gay G. \& Debaud J.C. (1988) Genetical variability of glutamate dehydrogenase activity in monokaryotic and dikaryotic mycelia of the ectomycorrhizal Hebeloma cylindrosporum. Appl. Microbiol. Biotech. 28, 566-571

Woolhouse H.W. (1969) Differences in the properties of the acid phosphatases of plant roots and their significance in the evolution of edaphic ecotype. In: Ecological Aspects of the Mineral Nutrition of Plants. Robinson R.M., éd., pp. 357-382 